\title{
Synchronous Pancreatic Ductal Adenocarcinoma and Hepatocellular Carcinoma: Report of a Case and Review of the Literature
}

\author{
JENNY Z. LAI ${ }^{1,2}$, YIHUA ZHOU ${ }^{3}$ and DENGFENG CAO ${ }^{2}$ \\ ${ }^{1}$ University College, Washington University in St. Louis, St. Louis, MO, U.S.A.; \\ ${ }^{2}$ Department of Pathology and Immunology, \\ Washington University in Saint Louis School of Medicine, St. Louis, MO, U.S.A.; \\ ${ }^{3}$ Department of Radiology, University of Pittsburgh School of Medicine, Pittsburgh, PA, U.S.A.
}

\begin{abstract}
Two or more histologically distinct malignancies diagnosed during the same hospital admission are uncommon, but they do exist. Cases with synchronous primary pancreatic ductal adenocarcinoma and hepatocellular carcinoma are rarely seen. This is a case report of a 56 years old Caucasian female with the chief complaint of jaundice over a duration of 10 days. CT imaging findings revealed a $3.5 \mathrm{~cm}$ ill-defined pancreatic head mass and a $1.5 \mathrm{~cm}$ liver mass in the segment 5. EUS FNA cytology showed pancreatic head ductal adenocarcinoma (PDAC). Liver biopsy revealed a well differentiated hepatocellular carcinoma (HCC). The patient underwent a pancreaticoduodenectomy and the pathology revealed a pancreatic ductal adenocarcinoma extending into peripancreatic soft tissue, portal vein and vascular groove with perineural invasion. This is a unique and challenging case with the coexistence of a primary PDAC and a primary HCC. To the best of our knowledge, this is the first documented case of synchronous PDAC and HCC in the English literature. The diagnosis and treatment of the two entities are discussed.
\end{abstract}

Synchronous cancers are defined as two or more primary cancers diagnosed in the same patient at the same time or within 6 months of each diagnosis $(1,2)$. Pancreatic ductal

Correspondence to: Dengfeng Cao, MD, $\mathrm{PhD}$, Department of Pathology and Immunology, Washington University School of Medicine, 660 S Euclid Ave Campus Box 8118, St. Louis, MO 63110, U.S.A. Tel: +1 3147475007, Fax: +1 3143628950, e-mail: dengfengcao@wustl.edu

Key Words: Synchronous carcinomas, hepatocellular carcinoma, pancreatic ductal adenocarcinoma, endoscopic ultrasound guided fine needle aspiration (EUS-FNA). adenocarcinoma (PDAC) is one of the fatal cancers with short-term survival rates. The case fatality rate for the disease is approximately $97 \%$ and has declined slightly (from 99\% to 97\%) in the last 12 years (3). To date, the causes of pancreatic cancer are not thoroughly understood, though certain risk factors such as smoking, obesity, lifestyle, diabetes mellitus, alcohol, dietary factors and chronic pancreatitis have been proposed. Hepatocellular carcinoma (HCC) is the second leading cause and the fastest rising cause of cancer-related death worldwide (4). One of the most common risk factors is cirrhosis due to multiple etiologies including viral infection, alcoholic or non-alcoholic steatohepatitis, and metabolism diseases. It is still challenging to obtain an early diagnosis of patients with PDAC or HCC. The patients with both devastating diseases diagnosed in the same hospital admission is very unusual. We herein report this case of a 56 years old Caucasian female who was diagnosed to have a PDAC and an incidentally identified well-differentiated HCC during the same diagnostic process. To the best of our knowledge through PubMed search, this is the first documented case of synchronous PDAC and HCC in the English literature. The diagnosis and treatment of the two entities are discussed.

\section{Case Presentation}

This is an outside consultation case with a recent diagnosis of pancreatic head ductal adenocarcinoma and hepatocellular carcinoma at an outside hospital. The patient was a 56 yearold Caucasian female with a chief complain of painless jaundice for 10 days. Patient also stated that she had experienced weight loss, pruritus, dark urine and clay colored stools, occasional nausea and some fatigue. Her past medical history included chronic diarrhea, anxiety and depression, occlusive thrombus, bleeding disorder, phlebitis and bone fracture. Chronic diarrhea, anxiety and depression 

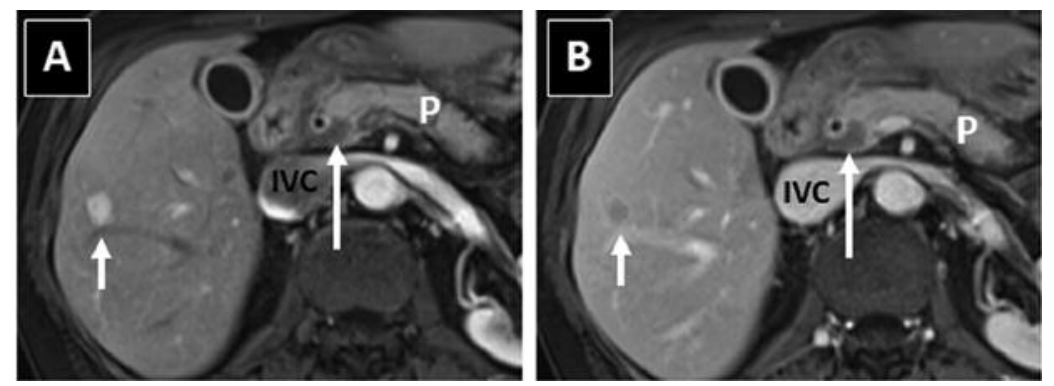

Figure 1. Post-contrast MRI images showing the liver and pancreatic masses. The post-contrast T1 weighted (T1WI) MRI images show hypoenhancing mass ( $A$ and $B$, long white arrow) in the head of the pancreas $(P)$ and a $1.5 \mathrm{~cm}$ mass $(A$ and $B$, short white arrow) in the liver. The pancreatic mass encases the common bile duct (the black dot). The liver mass demonstrates intense enhancement in the arterial phase (A) and mild washout in the venous phase $(B)$.

were present particularly for the past 18 months. Her social history included smoking (1/2 pack per day) and occasional alcohol consumption. Her lab tests showed a total bilirubin level of $17 \mathrm{mg} / \mathrm{dl}$ with predominant conjugated bilirubin. Other lab tests were unremarkable. She was clinically diagnosed as having painless obstructive jaundice. MRI and CT imaging findings showed an ill-defined pancreatic head mass with associated ductal stricture and a hepatic nodule in the segment 5 (Figure 1). An endoscopic ultrasound guided fine needle aspiration (EUS-FNA) and pancreatic ductal stricture brushing were performed. The cytology findings included clusters of hyperchromatic cells with enlarged nuclei, irregular nuclear membrane, scant cytoplasm, overlapping nuclei and 3D architecture (Figure 2). Benign ductal cells were also seen (Figure 2). The cytopathology diagnosis was positive for ductal adenocarcinoma.

Radiologically, the liver showed a $1.5 \mathrm{~cm}$ wellcircumscribed nodule with intense enhancement in the arterial phase and mild washout in the venous phase (Figure 1) and focal nodular hyperplasia or metastatic pancreatic carcinoma could not be excluded. A liver core biopsy was performed and showed lesional hepatocytes with mild cytologic atypia, some areas with increased N/C ratio as compared to adjacent benign liver parenchyma and unpaired thick-walled vessels. Focal pseudoglandular architecture was present. No intralesional bile duct or ductular reaction was identified. The lesional tissue showed positive staining for HepPar1, diffuse sinusoidal staining pattern of CD34, weak positivity for glypican 3 and focal loss of reticulin, and absence of iron compared to the adjacent benign liver parenchyma (Figure 3). A diagnosis of well differentiated HCC was made. The background hepatic parenchyma showed cholestasis, mild iron deposition with no significant chronic hepatitis, steatosis or cirrhosis identified. The outside EUS-FNA cytology diagnosis of PDAC and liver biopsy diagnosis of well differentiated hepatocellular carcinoma was also confirmed at Washington University.

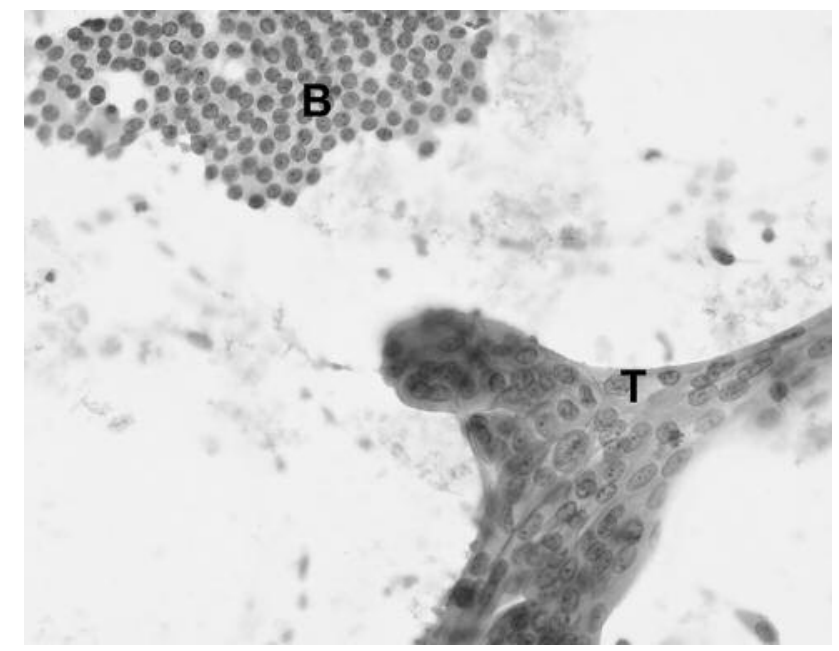

Figure 2. EUS-FNA Touch preparation showing clusters of ductal adenocarcinoma $(T)$ admixed with a few clusters of normal ductal epithelium (B) (Diff Quick stain x400).

After a multidisciplinary tumor board discussion of this case, the patient underwent a pancreaticoduodenectomy with lymph nodes dissection and the final pathology diagnosis was a $3.5 \mathrm{~cm}$ invasive moderately to poorly differentiated PDAC with extension into the portal vein and vascular groove and numerous foci of perineural invasion (Figure 4). Three of 18 lymph nodes were positive for metastatic PDAC. The pathologic tumor stage was pT3N1. The patient was given 4 cycles of FOLFIRINOX chemotherapy. During the six-month follow up, the patient did not feel well with chemotherapy and the liver nodule of the well differentiated HCC remained unchanged in size. During the one-year follow up, the patient was on palliative care and in the process of starting hospice. 


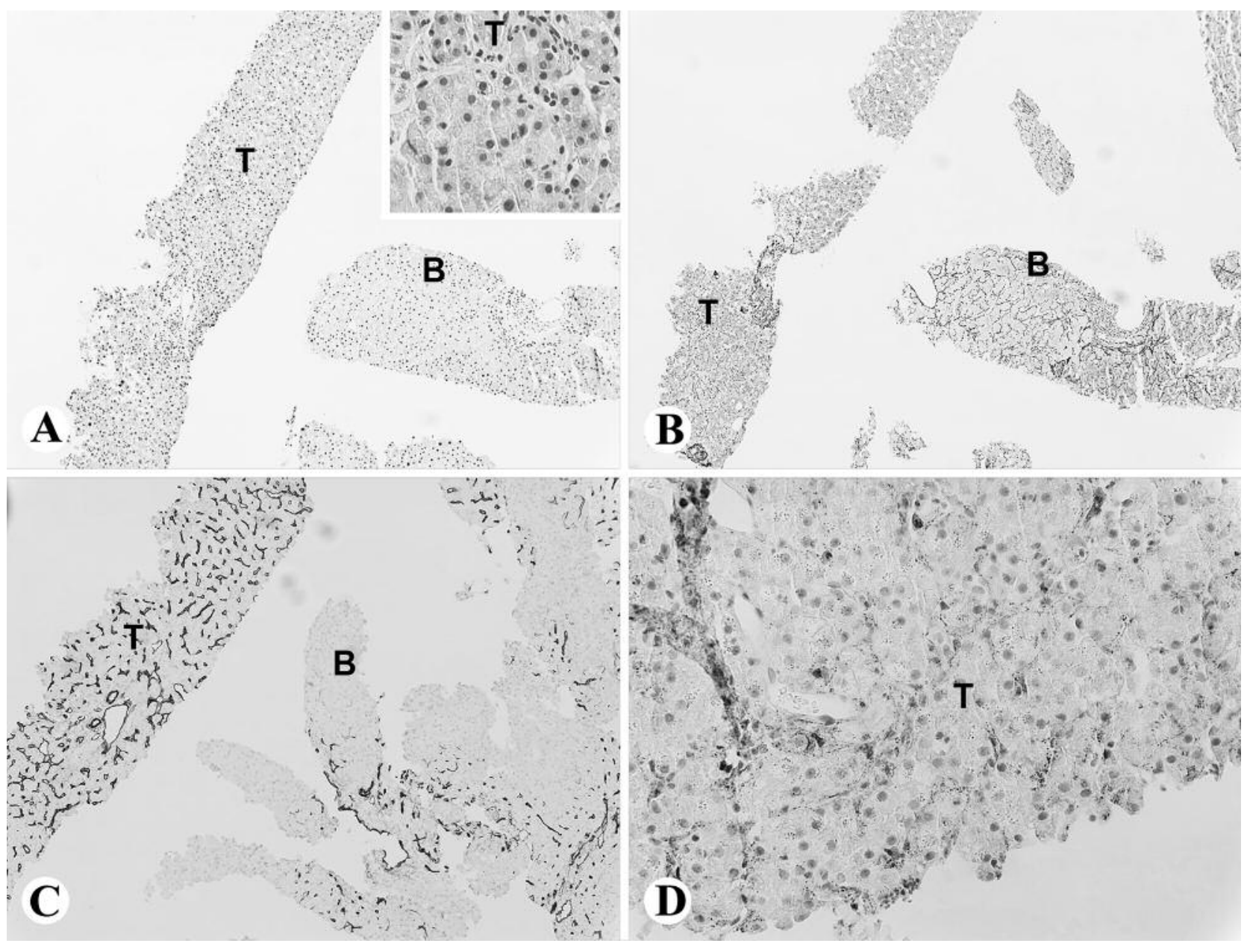

Figure 3. Hepatocellular carcinoma (HCC). A-D, liver biopsy showing well differentiated HCC (T) with mild cytologic atypia (A), focal loss of reticulin $(B)$, diffuse sinusoidal immunoreactivity of $C D 34(C)$ and weakly positive for glypican $3(D)$ compared to adjacent benign hepatic parenchyma $(B)$. (A-C, magnification $\times 100 ; D, \times 400)$.
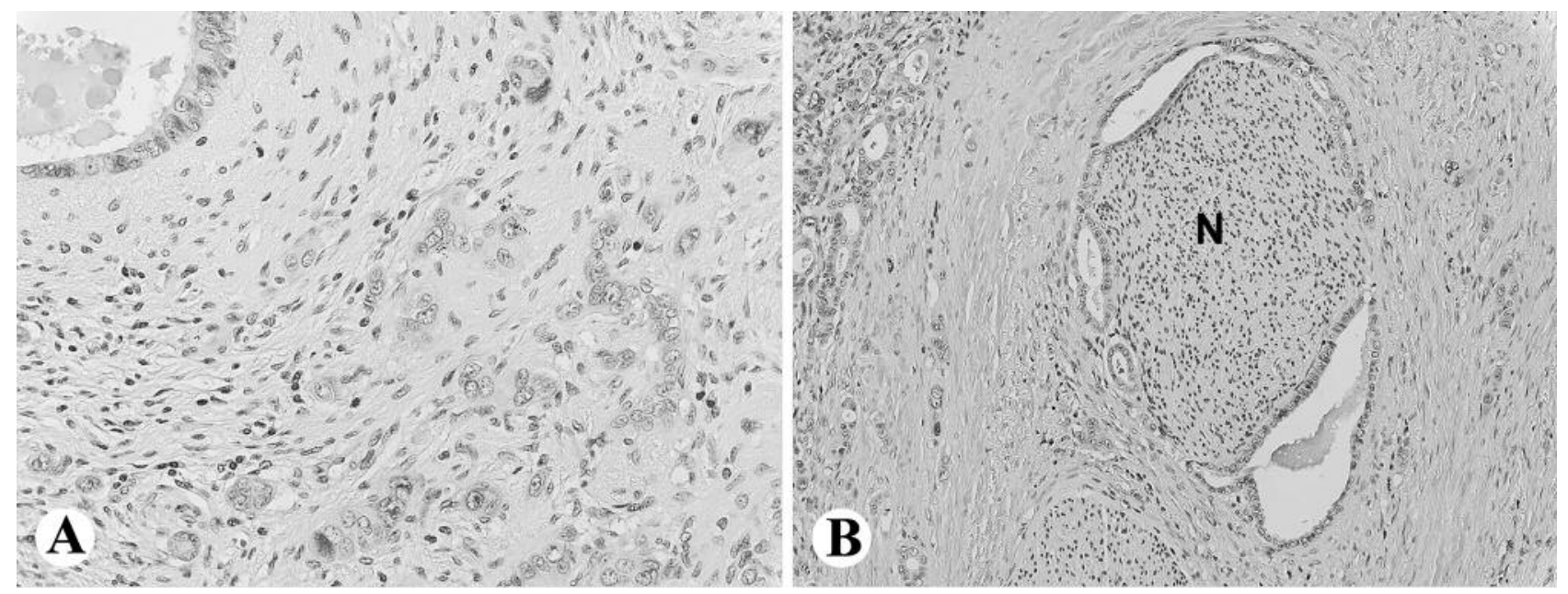

Figure 4. Partial pancreatectomy specimen showing moderately to poorly differentiated ductal adenocarcinoma with perineural invasion $(N)(H \& E$ stain, A, magnification $\times 400 ; B, \times 200)$. 


\section{Discussion}

Pancreatic cancer is the fourth leading cause of cancer death in the USA $(3,5)$. Despite developments in detection and management of pancreatic cancer, the survival rate is only $7 \%$ at 5 years post diagnosis. This low survival rate is partly a result of $80-85 \%$ of patients being diagnosed with advanced, unresectable disease (6). Thus, successful therapy depends significantly on early diagnosis (7). EUS-FNA has emerged as the primary modality for cytopathologic confirmation of PDAC, especially for smaller lesions $(<3 \mathrm{~cm})(8)$. It is also useful in patients with obstructive jaundice and no mass or an atypical/questionable mass seen on CT (7). The sensitivity of EUS-FNA for solid pancreatic masses ranges from $64-96 \%$ in meta analyses, with a specificity of over $95 \%(8,9)$. In this case, the patient had some unspecific symptoms including anxiety and depression but only had 10 days of painless obstructive jaundice that led to the diagnostic imaging study and EUS-FNA procedure. The EUS-FNA cytology diagnosis of PDAC was further confirmed by later pancreaticoduodenectomy.

The synchronous well-differentiated hepatocellular carcinoma was an incidental finding in this unique case. The imaging findings were also suspicious for metastatic PDAC or a benign process of focal nodular hyperplasia. The liver biopsy excluded these possibilities and showed the features of well differentiated HCC. Due to morphological differences, it is not difficult to distinguish primary HCC from liver metastatic PDAC, except in rare cases of PDAC with hepatoid features. There were no hepatoid features on this PDAC. Sometimes it's extremely difficult to distinguish a well differentiated HCC from primary benign liver nodules such as hepatocellular adenoma or focal nodular hyperplasia. In the present case, the features of tumor architecture showing unpaired thick-walled arteries and thickened hepatocyte plates with focal loss of reticulin framework, mild cytological atypia, absent iron deposition (compared to the adjacent benign parenchyma), diffuse sinusoidal staining pattern of CD34 and positive glypican 3 make the diagnosis of well differentiated HCC.

Treatment of the synchronous PDAC and HCC in this case is also very challenging since there are no similar reported cases seen in the literature. Based on multidisciplinary tumor board discussion of this case, a final decision was made to treat the PDAC first. The patient underwent a pancreaticoduodenectomy with lymph nodes dissection. The tumor pathologic stage was pT3N1 with three of eighteen lymph nodes positive for metastatic PDAC. The tumor also invaded the portal vein and vascular groove with numerous foci of perineural invasion. The patient was also given chemotherapy. In post chemotherapy, the well differentiated HCC remained unchanged in size. On one-year follow-up, the patient was on palliative care.
In summary, we report a rare and the first documented case of synchronous primary PDAC and HCC. The HCC was incidentally identified in the process of diagnosing PDAC. Treatment of the coexisting primary pancreatic and hepatocellular carcinomas are challenging. Based on this case, the moderately to poorly differentiated PDAC is more aggressive than the well differentiated HCC. Owing to the significant morbidity and mortality of these two carcinomas, a better understanding of the natural course of the diseases, the imaging and pathology presentation, and associated risk factors is imperative.

\section{References}

1 Piao J, Friedman P, Siddiqui S, Veerapong J and Lai JP: Synchronous type 1 papillary renal cell carcinoma in a patient with rectal adenocarcinoma. Anticancer Res 36(9): 4821-4824, 2016.

2 Meeks MW, Grace S, Chen Y, Petterchak J, Bolesta E, Zhou Y and Lai JP: synchronous quadruple primary neoplasms: colon adenocarcinoma, collision tumor of neuroendocrine tumor and schwann cell hamartoma and sessile serrated adenoma of the appendix. Anticancer Res 36(8): 4307-4311, 2016.

3 Ilic M and Ilic I: Epidemiology of pancreatic cancer. World J Gastroenterol 22: 9694-9705, 2016.

4 Ferlay J, Soerjomataram I, Dikshit R, Eser S, Mathers C, Rebelo M, Parkin DM, Forman D and Bray F: Cancer incidence and mortality worldwide: sources, methods and major patterns in GLOBOCAN 2012. Int J Cancer 136(5): E359-386, 2015.

5 Siegel RL, Miller KD and Jemal A: Cancer statistics. CA Cancer J Clin 2015(65): 5-29, 2015.

6 Vincent A, Herman J, Schulick R, Hruban RH and Goggins M: Pancreatic cancer. Lancet 378: 607-620, 2011.

7 Lai JP, Yue Y, Zhang W, Zhou Y, Frishberg D, Jamil LH, Mirocha JM, Guindi M, Balzer B, Bose S, Cao D, Lo S, Fan X and Rutgers JK: Comparison of endoscopic ultrasound guided fine needle aspiration and PET/CT in preoperative diagnosis of pancreatic adenocarcinoma. Pancreatology 17(4): 617-622, 2017.

8 Hewitt MJ, McPhail MJ, Possamai L, Dhar A, Vlavianos P and Monahan KJ: EUS-guided FNA for diagnosis of solid pancreatic neoplasms: a meta-analysis. Gastrointest Endosc 75: 319-331, 2012.

9 Puli SR, Bechtold ML, Buxbaum JL and Eloubeidi MA: How good is endoscopic ultrasound-guided fine-needle aspiration in diagnosing the correct etiology for a solid pancreatic mass? A meta-analysis and systematic review. Pancreas 42: 20-26, 2013.

Received March 10, 2018

Revised March 27, 2018

Accepted March 28, 2018 